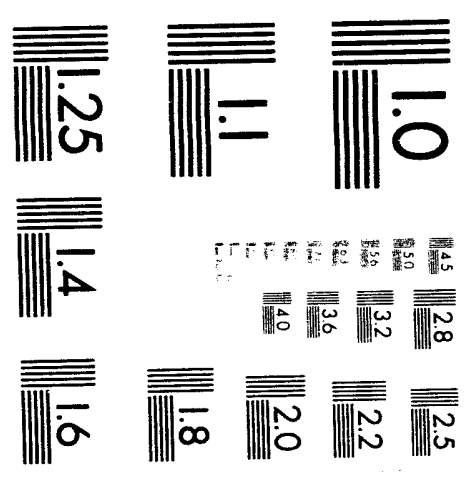



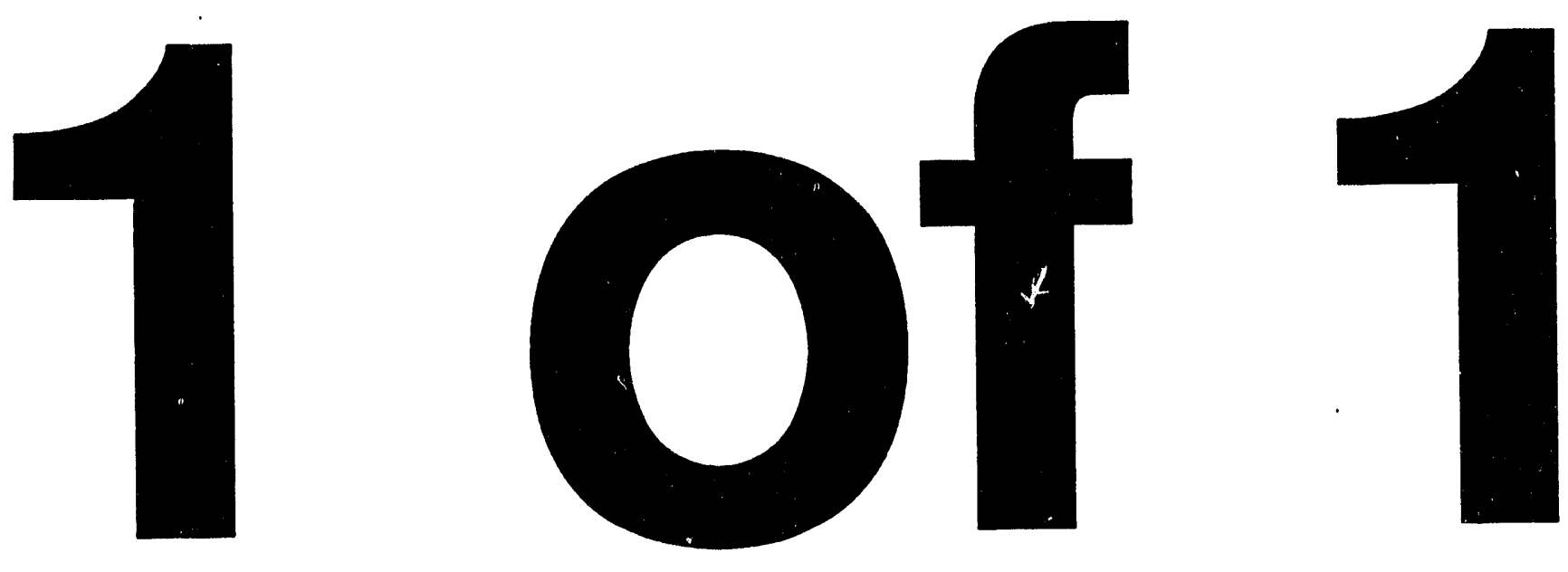
GA-A21423

\title{
DIII-D NEUTRAL BEAM CONTROL SYSTEM OPERATOR INTERFACE
}

by

\author{
J.J. HARRIS and G.L. CAMPBELL
}

This is a preprint of a paper to be presented at the 15th IEEE/NPSS Symposium on Fusion Engineering, October 11-15, 1993, in Hyannis, Massachusetts, and to be printed in the Proceedings.

\author{
Work supported by \\ U.S. Department of Energy \\ Contract DE-AC03-89ER51114
}

\section{GENERAL ATOMICS PROJECT 3466 \\ OCTOBER 1993}

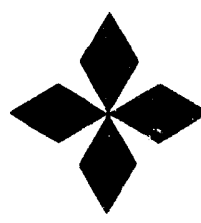

\section{GeNERAL}

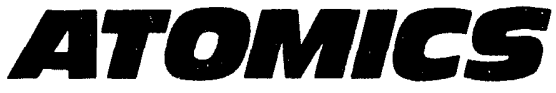




\title{
DIII-D NEUTRAL BEAM CONTROL SYSTEM OPERATOR INTERFACE*
}

\author{
J.J. Harris and G.L. Campbell \\ General Atomics \\ P.O. Box 85608, San Diego, California 92186-9784
}

\begin{abstract}
A centralized graphical user interface has been added to the DIII-D Neutral Beam (NB) control systems for status monitoring and remote control applications. This user interface provides for automatic data acquisition, alarm detection and supervisory control of the four NB programmable logic controllers (PLC) as well as the Mode Control PLC. These PLCs are used for interlocking, control and status of the NB vacuum pumping, gas delivery, and water cooling systems as well as beam mode status and control. The system allows for both a friendly user interface as well as a safe and convenient method of communicating with remote hardware that formerly required interruption of machine operations to access. In the future, to enable high level of control of PLC subsystems, complete procedures can be written and executed at the touch of a screen control panel button. The system consists of an IBM compatible 486 computer running the FIX DMACS ${ }^{\mathrm{TM}}$ for Windows ${ }^{\mathrm{TM}}$ data acquisition and control operator interface software, a Texas Instruments/Siemens communicatio' card and Phoenix Digital optical communications modules. Communication is achieved via the TIWAY (Texas Instruments) protocol link utilizing both fiber optic communications and a copper local area network (LAN). Hardware and software capabilities will be reviewed. Data and alarm reporting, extended monitoring and control capabilities will also be discussed.
\end{abstract}

\section{INTRODUCTION}

DIII-D is a magnetic confinement fusion device operated by General Atomics for the U.S. Department of Energy. Four beamlines, each with two common long pulse sources, are used to provide auxiliary plasma heating [1]. A Control Instrumentation $(\mathrm{CI})$ rack, located adjacent to each neutral beam tank, controls the vacuum, gas delivery and water cooling systems for each beamline. The $\mathrm{Cl}$ also provides monitor points for alarm reporting, interlocking; power supply permits and control computer interface. Also vital to the control, monitoring and interlocking of neutral beam systems is the Mode Control System [2]. While Mode Control functions primarily as an interface to select and fire several different types of beam shots, it is also an integral part of the status monitoring and interlock scheme for beam operations.

The heart of both the CI stations and the Mode Control are Texas Instruments (TI) programmable logic controllers (PLC). These systems were upgraded several years ago with the intent that they would be later networked and linked to a host interface station [3]. In 1991, network hardware was purchased and installed to provide a high speed, dedicated communication link to the PLCs. The network was primarily used for program loading, ladder logic status monitoring and diagnostics. At that time, operator interface SCADA (Supervisory Control and Data Acquisition) packages did not offer features such as object oriented graphics, advanced V/O device drivers and cross platform software, in one package for an IBM PC compatible computer.

In mid-1992 The FIX DMACS ${ }^{\mathrm{TM}}$ (Fully Integrated Distributed Manufacturing Automated Control System) SCADA package for Windows ${ }^{\mathrm{TM}}$ became available. Although an 1/O device driver was not available for the TI PLC, Intellution Inc. developed the driver for us. The $\mathrm{I} / \mathrm{O}$ device driver "talks" to the remotely located PLC hardware via the high speed protocol network. The FIX DMACS ${ }^{\mathrm{TM}}$ met all of the criteria for our application. Database creation is made easy because of the spreadsheet style of the Database Builder. Graphical presentation is achieved using the object oriented Display Builder or by importation of AutoCAD files. Process automation, historical trending, event and alarm logging were also important features for our application. Process monitoring and control; alarm and event logging for all NB control systems is now accomplished using a 486DX central control computer (Host) linked via fiber optics to the remotely located hardware. Critical real time valve control, status and interlock condition information is now available to operators during experimental periods. During non-operation periods data collected can be exported to spreadsheets or other analysis programs to be archived and analyzed.

\section{COMMUNICATION HARDWARE DESCRIPTION}

PLC network communication is implemented with a variety of hardware (Fig. 1). A TI Unilink Host Adapter (Unilink) card is the key to directing network traffic. The Unilink is a bus resident card located in the Host. Local/line NIMs (Network Interface Module) which talk to the Unilink, reside in each PLC crate. Each NIM has a unique address on the network. The Unlink and NIMs are tied together in a master/slave fashion on the TIWAY I protocol network which uses both a multi-drop copper LAN, as well as an optical communication (OCM) interface which is PLC buss resident. OCMs have a master/slave relationship with each other on this particular network to prevent PLCs from inadvertently sending commands to each other. The fiber optics prevent

\footnotetext{
*Manuscript received October 11, 1993. This work was supported by the U.S. Department of Energy under Contract No. DE-AC03-89ER51114.
} 


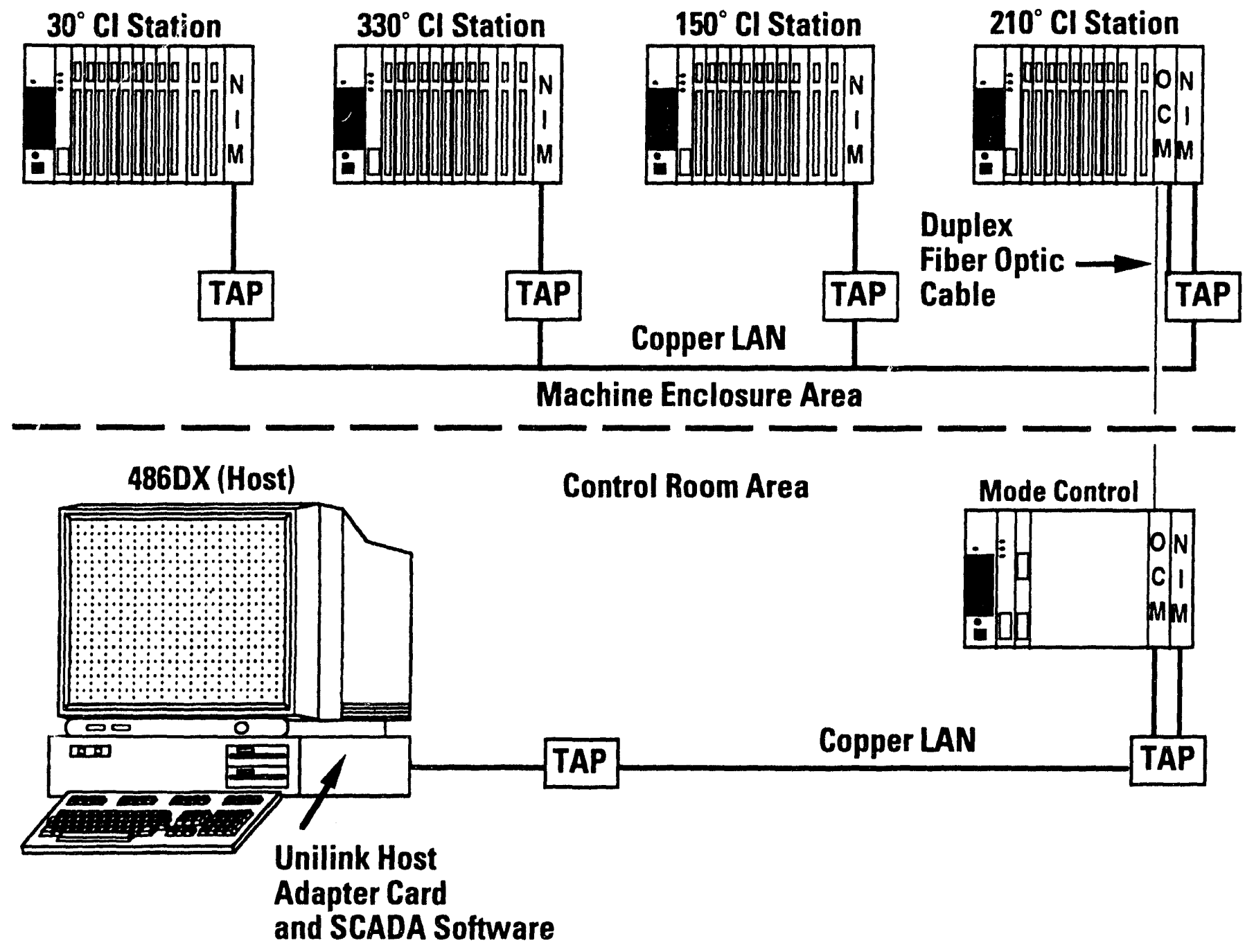

Fig. 1. Neutral beam control system PLC network communication block diagram.

ground loop problems and provide the $30 \mathrm{kV}$ electrical isolation required. Communication between NIMs in the remote area takes place on a multi-drop copper network consisting of a main trunk cable and droplines for each PLC. We chose to use the copper LAN in the remote machine enclosure area in order to save costs. Although RFI and EMI are present in the machine enclosure area, we did not need to use the NIM redundant port capability. Communication on the network has been very reliable.

The Unilink has several functions. The Unilink reads and writes data to the PLC (via the NIM). It also manages communication tasks by arbitrating all communication activity on the network. Read/write requests (from the program loader and the SCADA software packages) are converted to network commands and temporarily stored for later task completion verification. If there is no response within a preset window an error message is received.

The NIM has three functions (Fig. 2). The first is to deliver and receive PLC communication. The second function is to translate the communication between PLC command language and NIM Primitives. The third function of the NIM is to supervise transactions between it and the Unilink. NIMs com- municate with the Unilink asynchronously at 115,200 baud using HDLC protocol and NRZI encoding. NIMs can be taken off-line via a local/remote switch located on the front panel of the NIM. When the NIM is in local mode the Host cannot write data to the PLC, however, data can still be read. This function is especially useful during maintenance and specialized testing periods when valve control needs to be isolated to the local site.

Unilink and NIM information is exchanged using NIM Primitives. Primitives are high level commands that allow the user to access like data types in different PLCs in the same manner. Use of these primitives help to reduce the amount of host computer software needed to gather data from the PLC. Primitives allow more efficient use of the network bandwidths thereby reducing system loading. The NIMs can "memorize" data locations that are repeatedly accessed, thereby making information transfer that much faster.

The FIX DMACS ${ }^{\mathrm{TM}}$ SCADA package communicates only with the Unilink over the Host buss. The communication between the FIX I/O device driver and the Unilink will be explained in the next section. 


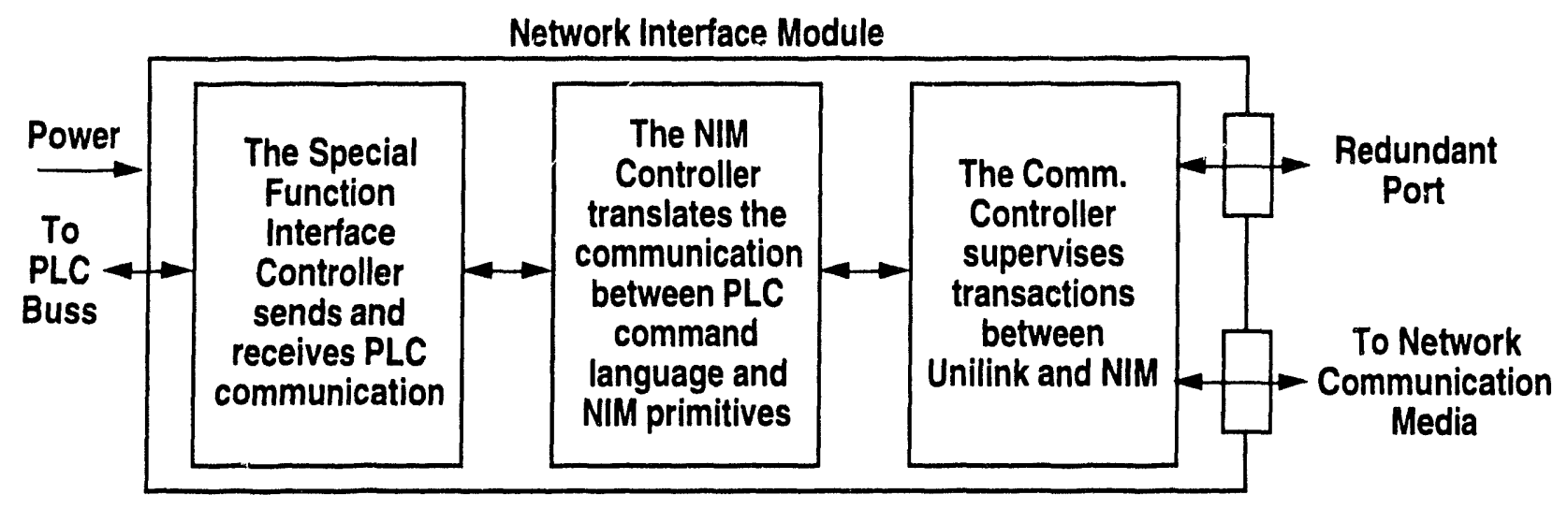

Fig. 2. NIM functional diagram.

\section{SCADA SOFTWARE CAPABILITY}

The FIX DMACS ${ }^{\mathrm{TM}}$ for Windows ${ }^{\mathrm{TM}}$ provides the key elements of the automation system; configuration, data collection and analysis, operations and reporting (Fig. 3). First we will look at configuration.

The System Configuration Utility (SCU) is the basic building block for running and directing the SCADA system traffic. Information paths are established for saving files and directing where alarms, operator messages and historical collection will end up. The SCU also establishes connections between the database and the $1 / O$ device driver. Configuration of this driver is also accomplished here using the Driver Image Definition program. It is within the SCU that tasks such as 1/O driver loading, SAC (Scan, Alarm and Control) and View (runtime) are configured and loaded during the startup routine.

The Database Builder (DBB) configures read/write $\mathrm{V} / \mathrm{O}$ blocks which manipulate data to and from the control strategy during runtime. The DBB is a menu driven, spreadsheet like program that uses engineering terms to define database variables. Information such as $\mathrm{V} / \mathrm{O}$ address (PLC node and $\mathrm{I} / \mathrm{O}$ point), a unique tagname, alarm and security parameters, and scan times are included in each DBB point. The DBB information can also be stored in ASCII form and exported to a

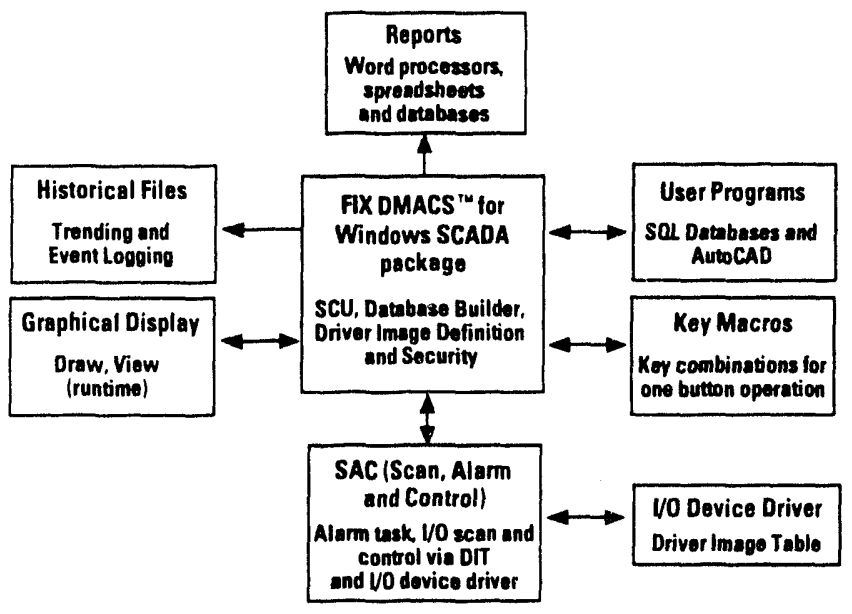

Fig. 3. Key elements and interaction of the SCADA package spreadsheet or word processing program. Database items can be sorted, queried and displayed in a number of ways. Additionally, database changes can be made on-line while still controlling the process.

The next part of the configuration process takes place in Draw. This is an object oriented drawing program where runtime display screens can be created. AutoCAD displays can also be imported in and attached to database points for runtime screens. Bar and trend graphs, flashing, pop-up and audible status's, alphanumeric data entry, alarm queues and operator advisories are all configurable under Draw. Draw pictures are used for operator interface control and status during runtime.

Security levels are configurable by establishing individual and group user accounts that allow or restrict access to database block writes, specific programs and operator displays. Once user accounts have been established one must log on to the system using a name and password.

Data handling is done in several steps. The $\mathrm{I} / \mathrm{O}$ device driver gets its information only from the Unilink card, it then uses the driver image definition created earlier in the SCU to load a Driver Image Table (DIT) during start-up. The I/O device driver refreshes the DIT continuously based on scan times defined in the DBB. SAC then uses the information from the DIT to update View screens and other tasks.

\section{SCADA APPLICATION, CONFIGURATION AND FUNCTION}

At the present time a single SCADA node is being used for both program development during maintenance periods and for operations support during experimental periods. The runtime display screens have been developed based on the information operators need during specific types of experiments. For example, an Injection Status page was created for use when any neutral beam injector is being using for heating the plasma of the DIII-D tokamak. Vacuum and water status, cryo and power supply permit information for all four beamlines are included on this page. Operators can tell at a glance if there are abnormal conditions. The operators may then go to the screen specific to the subsystem affected for detailed information and to provide a remedy. There are a number of 
other pages that show both summary and detail information about the four NB CI stations and Mode Control systems. Screens with complete ion source and heamline water status information have been developed. Screens for vacuum and gas system control, status; and alarm reporting have also been designed. Additionally, specialized screens have been developed for experiments that require unusual monitoring and control. For example, during a recent reactive gas fueling experiment, the plasma was fueled with diborane gas and pumped out using 2 of the neutral beam injectors. A special page was created in a small amount of time allowing the operators to monitor and control specific portions of the vacuum system during that experiment.

Pages have been created to imitate the control panels located in the $\mathrm{CI}$ stations at the remote site. This has been done initially to make the transition from hardware to computer interface go more smoothly providing the operators with the same 'look' that they are used to. These screens will evolve into something more complex in the future as complete processes can he performed at the touch of a screen button. Much of the complexity will be programmed at the development level and will be invisible to the user.

\section{OPERATING EXPERIENCE AND FUTURE PLANS}

Significant effort has been devoted to creating the large database from which to create and update the screens. Each I/O point used contains information that is used by different portions of the SCADA package. There are 5 PLCs presently on the network each with approximately $512 \mathrm{~V} / \mathrm{O}$ addresses to read or write to. However, it is not necessary to monitor or control each point. Some portions of the PLC ladder logic programs have been changed in order to reduce the amount of traffic on the network thus allowing for faster response and screen updates.

We are still in the process of making better use of the many features of the SCADA package. For example, Historical Trending has been recently added. This allows us to log analog data (beamline pressure, for example) and events. Event logging can help us keep track of occurrences such as each time a valve is cycled or perhaps each time a control breaker is opened or closed. Historical data can be stored in snapshot form (trend) or log form (event) for later manipulation, archival and report generation.

Another desirable feature of the SCADA package is Key Macros. Using this option enables the programmer to customize the environment by attaching variables to command(s). For example, if you are viewing a summary page and a variable changes or alarms, the operator can click on that variable and a detailed screen will pop up. We will make extensive use of macros to implement process control at the touch of a button.

The DDE (Dynamic Data Exchange) server is another useful tool allowing exportation of files (especially archival) to spreadsheets, databases and word processing programs. This feature is especially useful for report generation.

Our experience with this system nas been both good and bad. The hardware performance has been flawless. However, there were initial problems with getting the $\mathrm{I} / \mathrm{O}$ device driver working properly. We are especially pleased with the ease with which all of the SCADA definition is accomplished. Almost all of the pieces of the package (DBB, DID, Display Builder etc.) are straightforward, menu driven programs which makes additions and changes to the database relatively easy. This is especially convenient hecause as we utilize more features, and continually add to the database, the task is not so burdensome. Input from operators and other users has been helpful is developing a system that is both useful and easy to use. Changes are continually being made to create a friendly user environment.

\section{CONCLUSION}

Improved operational flexibility has been gained from the centralized graphical user interface for the neutral beam control system. All PLC data can now be controlled and monitored from one place for both real-time experimental application and later analytical and archival use. This information is presented in an easy to understand graphical window environment. We can also incrementally add functionality and increase performance without stopping the process. Finally, decisions at the operator level can be minimized (or enhanced) depending on the level of control required. The network has proved to be rugged and reliable and can be used for PLC ladder program loading, diagnostics and SCADA operation from a single IBM PC compatible platform. The cross platform capabilities extend to both creation and maintenance of the database and viewing screens as well as archival data reporting. This new system provides complete control from a single platform and has reduced the need for using unfriendly ladder logic status monitoring and need for machine enclosure access during experimental periods.

\section{ACKNOWLEDGMENT}

We thank the Neutral Beam Operations group for their enthusiastic support throughout this project.

\section{REFERENCES}

(1) Colleraine, A.P., D.W. Doll, R. Hong, J. Kim, A.R. Laznghom, C.H Meyer, et al., "The DIII-D long pulse neutral beam system," in Proc 11th Symposium on Fusion Engineering. (198.5) pp. 1278- 1281.

(2) Kohli, J.C., J. Garcia, A. Glad, J. Haskovec, C.D. Moore, J. Tooker, et al.. "Neutral beam mode control," in Proc. 9th Symposium on Engineering Problems of Fusion Research. (1981) pp. 2058-2061.

[3] Gilgallon, J.J. and J. Phillips, "Control system upgrade of the DIII-D neutral beamline," in Proc. 13th Symposium on Fusion Engineering. (1989) pp. 175-177 

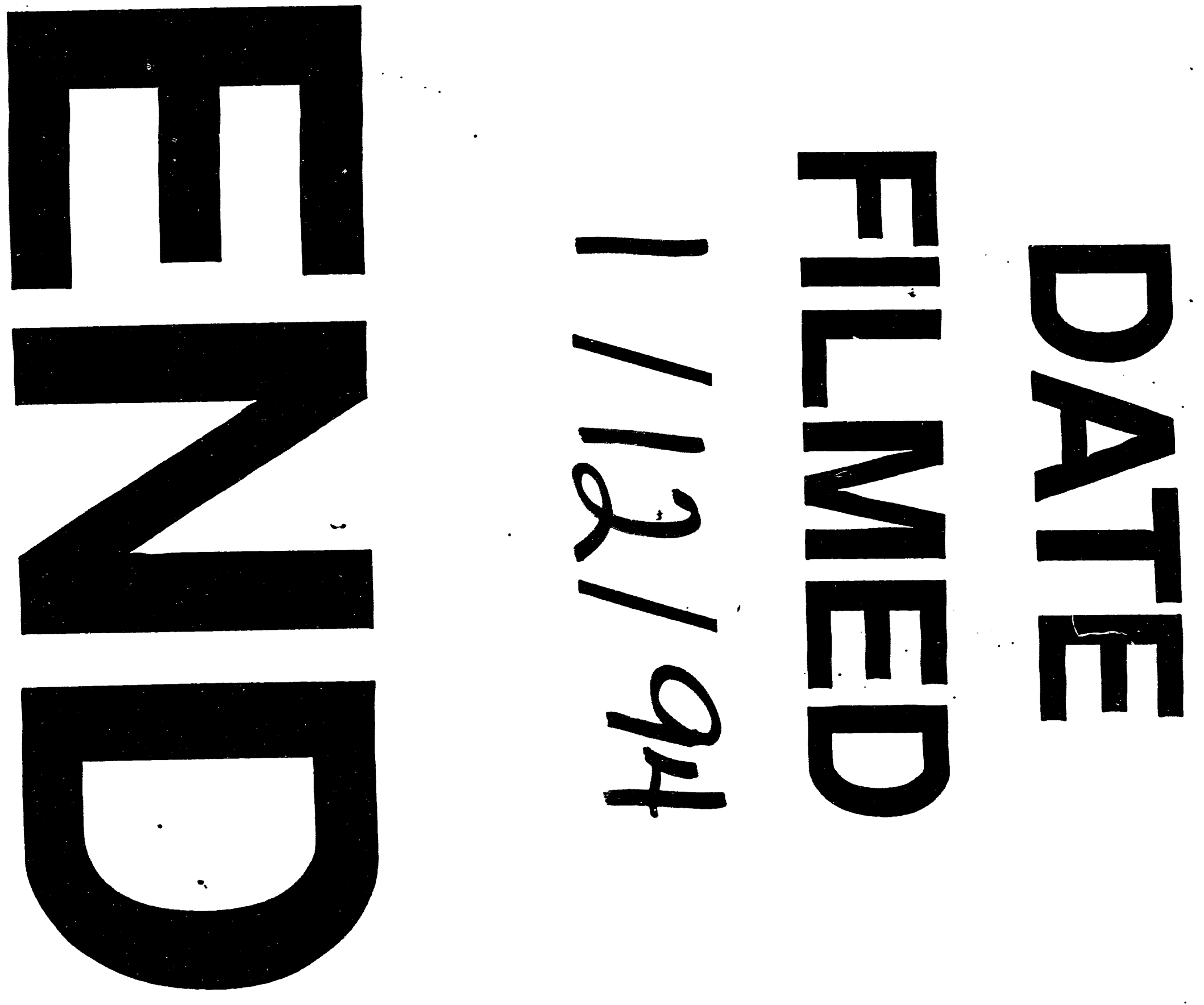
